\title{
Torque de remoção de parafusos em pilares protéticos com conexão tipo hexágono interno após ciclagem mecânica
}

\author{
Evaluation of the torque removal in internal hexagonal abutments submitted to a cyclic \\ loading test
}

Patrícia Maria RANGEL

Estagiária da Disciplina de Prótese Parcial Fixa - Faculdade de Odontologia de São José dos Campos - Universidade Estadual Paulista - UNESP - São José dos Campos - SP - Brasil

\section{Gabriel Portela PAULO}

Estagiário da Disciplina de Prótese Parcial - Faculdade de Odontologia de São José dos Campos - Universidade Estadual Paulista - UNESP - São José dos Campos - SP - Brasil

\section{Marianna Cavalheiro GONÇALVES}

Mestranda em Odontologia Restauradora - Área de Concentração em Prótese - Faculdade de Odontologia de São José dos Campos - Universidade Estadual Paulista - UNESP - São José dos Campos - SP - Brasil

\section{Marcos Koiti ITINOCHE}

Mestre e Doutor em Odontologia Restauradora - Área de Concentração em Prótese - Faculdade de Odontologia de São José dos Campos - Universidade Estadual Paulista - UNESP - São José dos Campos - SP - Brasil

\section{Fernando Eidi TAKAHASHI}

Professor Assistente Doutor - Disciplina de Prótese Parcial Fixa - Faculdade de Odontologia de São José dos Campos Universidade Estadual Paulista - UNESP - São José dos Campos - SP - Brasil

\section{Renata FARIA}

Mestre e Doutor em Odontologia Restauradora - Área de Concentração em Prótese - Faculdade de Odontologia de São José dos Campos - Universidade Estadual Paulista - UNESP - São José dos Campos - SP - Brasil

\section{Resumo}

O sucesso no tratamento reabilitador protético realizado sobre implantes ósseo-integrados pode ser comprometido por falhas mecânicas em seus componentes, tais como desajuste e até mesmo fraturas dos componentes protéticos quando expostos à função mastigatória. O presente estudo teve por objetivo avaliar, in vitro, o desajuste ocorrido na interface implante-intermediário por meio da aferição e comparação do torque inicial utilizado para a colocação do parafuso de fixação dos pilares protéticos em implantes com hexágono interno, com relação ao torque necessário para remoção dessas estruturas antes e após a simulação da função mastigatória. Testes de ciclagem mecânica foram induzidos sobre os pilares protéticos. Mediu-se o torque de remoção dos parafusos de fixação dos pilares proteticos. Houve diminuição significativa nos valores de torque de remoção de todas as amostras, e a perda foi ainda maior nas amostras submetidas à ciclagem mecânica. Os dados obtidos permitem supor que há sempre diminuição nos valores de torque de remoção desses componentes e que a função mastigatória aumenta a probabilidade de desajuste na interface implante-pilar protético.

\section{UNITERMOS}

Implantes; componente protético, ciclagem mecânica, torque de remoção. 


\section{INTRODUÇÃO}

A Odontologia sempre buscou um material restaurador que possua características semelhantes ao dente natural, tanto do ponto de vista estético-funcional, como em relação à durabilidade e resistência ${ }^{1}$. Deve-se a isso, principalmente, o grande destaque que o uso de implantes ocupa na Odontologia atual. Implantes dentais permitem a reabilitação protética, com qualidade, sem comprometimento de outros elementos dentais sadios. O material comumente utilizado, o titânio, possui atributos tais como biocompatibilidade, boas propriedades mecânicas, resistência à corrosão e manchamento e custo acessível ${ }^{2}$. Implantes ósseointegrados de titânio apresentam, portanto, excelente comportamento frente aos tecidos ósseo e mucoso, fator determinante no sucesso desse tipo de tratamento restaurador.

A estabilidade das próteses sobre implantes, numa análise longitudinal, está relacionada à adaptação passiva dessas estruturas ${ }^{3,4}$. É essencial que haja precisão na conexão entre implante e seus componentes, que devem ser usinados de forma cuidadosa para favorecer a adequada adaptação $0^{5}$. O uso de próteses sobre implantes parafusadas exige o retorno periódico do paciente ao consultório, o afrouxamento dos componentes provoca rotação da prótese, quando unitária, e são necessários reapertos ${ }^{6,7}$. Ainda, a longo prazo, podem ocorrer deformações nas roscas do parafuso com redução da capacidade de união das partes e necessidade de troca de tal estrutura. Há ainda outros fatores que afetam a estabilidade do sistema, como defeitos de fabricação, formas de resistência antirotacional e quantidade de lubrificante utilizado ${ }^{8,9,10}$, ou uma somatória de falhas, como repetidos apertos e desapertos, com desgaste friccional das roscas e alteração das estruturas, resultando ainda, em queda da resistência e perda do torque inicial quando o sistema está em função $0^{4}$. Além disso, a forma como o estresse mecânico é transferido do implante ao tecido ósseo adjacente, é fator determinante no êxito clínico do tratamento, visto que, se esse processo gerar forças de grande magnitude, há riscos de comprometimento da longevidade do implante e seus componentes ${ }^{11}$.

O controle clínico inicial da adaptação marginal do implante e seus componentes protéticos pode ser realizado de acordo com o material utilizado na infra-estrutura da prótese bem como o torque inicial utilizado na fixação dos componentes protéticos ${ }^{12}$. Sabe-se que os sistemas de implantes presentes no mercado apresentam bom nível de controle de quali- dade $^{5}$, mas pouco se sabe ainda sobre a influência do ato mastigatório na resistência mecânica da interface implante-pilar protético ${ }^{13}$, uma vez que, em função, podem apresentar desgaste e desajuste na união.

A função mastigatória fisiológica não deve ter influência na adaptação passiva entre implante e seus componentes protéticos. Para um estudo laboratorial, pode-se utilizar a ciclagem mecânica para simulação do ato mastigatório, de modo a aproximar o ensaio das condições normalmente encontradas no meio bucal ${ }^{14}$. $\mathrm{O}$ ensaio é realizado submetendo-se a estrutura a ser avaliada a ciclos repetitivos em meio aquoso, com intensidade, freqüência e temperatura controladas ${ }^{15}$.

Outro indicativo na qualidade da adaptação dos componentes protéticos aos implantes é o torque inicial utilizado para a fixação. Um estudo mostra que uma carga baixa no torque inicial $(16 \mathrm{~N} . \mathrm{cm})$ provoca grande desajuste na interface implante-pilar protético, quando comparado a amostras que receberam valores de carga mais altos $(32 \mathrm{~N} . \mathrm{cm} \text { e } 48 \mathrm{~N} . \mathrm{cm})^{16}$. Em microscopia óptica também se observou melhor adaptação em conexões que receberam $20 \mathrm{~N}$.cm de torque inicial em relação às que receberam 10 N.cm em cilindros de diferentes ligas metálicas ${ }^{1}$.

Por último, toma-se por referencial na resistência de união entre implantes e seus componentes protéticos a anatomia da conexão. Pilares com encaixes internos geralmente apresentam-se mais estáveis que pilares com encaixe externo ${ }^{8}$.

Dessa forma, o objetivo do presente estudo é avaliar quantitativamente a perda de torque inicial utilizado na fixação do pilar protético ao implante. Para tanto, mensurou-se o torque de remoção dos parafusos interconectores em amostras com implantes de hexágono interno e seus respectivos componentes protéticos submetidos à ciclagem mecânica.

\section{Materiais e mÉtodos}

Dez implantes de $3,75 \mathrm{~mm}$ de diâmetro x $10 \mathrm{~mm}$ de altura com hexágono interno (Conect AR - Conexão Sistemas de Prótese) foram incluídos, individualmente, em blocos cilíndricos de resina acrílica com $15 \mathrm{~mm}$ de altura $\mathrm{x} 10 \mathrm{~mm}$ de diâmetro com o longo eixo paralelo ao eixo central dos cilindros utilizando-se, para tal, um delineador (BioArt). Os pilares protéticos (Pilar de Preparo Munhão 128071 - 1mm - Conexão Sistemas de Prótese) foram conectados aos implantes por meio de seus respectivos parafusos de fixação com torque inicial de $30 \mathrm{~N}$, controlado com o auxílio de um torquímetro mecanico(Nobel Biocare). 
Em cinco amostras, procedeu-se imediatamente a remoção dos parafusos de fixação, com uso de um torquímetro analógico (Tohnichi BTG60CN) verificando-se os valores de torque de remoção dessas estruturas sem a ação de qualquer agente externo.

Para a realização dos ensaios, os demais corposde-prova foram submetidos, individualmente, a ação da cicladora mecânica artesanal (desenvolvida por Itinoche ${ }^{15}$ ).

Cada corpo-de-prova foi posicionado sobre uma base metálica com inclinação de $45^{\circ}{ }^{17}$. Nesta base, há um termostato que permitiu a realização do teste em meio aquoso, à temperatura constante de $37^{\circ} \mathrm{C}$. No equipamento, uma haste superior, fixada em uma pinça, foi usada para induzir impulsos de carga axial constante de $50 \mathrm{~N}^{18}$, com frequiência de $2 \mathrm{~Hz}$ por 50.000 ciclos sobre os pilares protéticos.

Após a ciclagem, procedeu-se a remoção dos parafusos de fixação dos componentes protéticos. $\mathrm{O}$ procedimento foi realizado novamente com auxílio do torquímetro digital para aferição da força necessária à remoção dos parafusos.

Para avaliar o relacionamento entre o torque de remoção dos parafusos dos pilares protéticos e a influência da ciclagem mecânica, os dados obtidos neste experimento foram submetidos ao modelo estatístico de amostras independentes com o Teste $t$ (Student).

\section{Resultados}

A estatística descritiva dos dados de torque de remoção dos parafusos de fixação dos pilares protéticos obtidos é apresentada na Tabela 1 e representada mediante o gráfico (Figura 1).

TABELA 1. Estatística descritiva dos dados de torque de remoção dos pilares protéticos (valores em N.cm) e de perda em cada amostra. Valor de torque inicial idêntico em todas as amostras (30N.cm).

\begin{tabular}{cc}
\hline Grupo Controle & $\begin{array}{c}\text { Grupo Pós Ciclagem } \\
\text { Mecânica }\end{array}$ \\
\hline $29(1)$ & $26(4)$ \\
\hline $29(1)$ & $24(6)$ \\
\hline $28(2)$ & $23(7)$ \\
\hline $27(3)$ & $18(12)$ \\
\hline $28(2)$ & $18(12)$ \\
\hline${ }^{*} n=5$ &
\end{tabular}

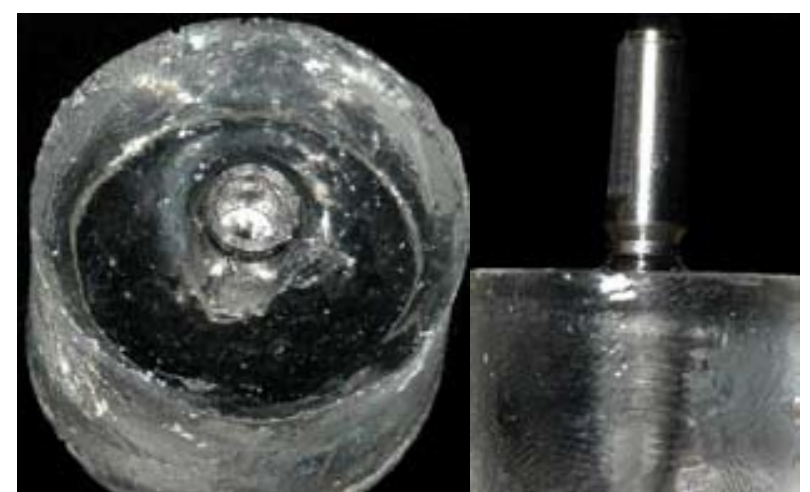

FIGURA 1 - Vista superior da amostra sem o pilar protético, e em perfil, já com o pilar em posição.

Verificou-se perda de torque em todas as amostras, dos dois grupos avaliados. A perda média de torque obtida nas condições: Controle $(1,80 \pm 0,84$ N.cm) e Pós Ciclagem Mecânica $(8,20 \pm 3,63 \mathrm{~N} . \mathrm{cm})$ diferem estatisticamente [t(Student $)=3,84]$.

Os dados quando submetidos ao teste $\mathrm{t}$ (Student) de amostras independentes, possibilitam rejeitar a hipótese de igualdade referente ao efeito controle (sem ciclagem mecânica) x pós-ciclagem mecânica, e ainda, ao efeito torque inicial x torque de remoção.

Por meio dos dados estatísticos obtidos, podemos fazer algumas considerações:

- Foi observada diferença estatisticamente significante entre os diferentes procedimentos aplicados a cada grupo avaliado (Controle e Pós Ciclagem Mecânica).

- A interação entre ciclagem mecânica e torque de remoção apresentou diferença estatisticamente significante.

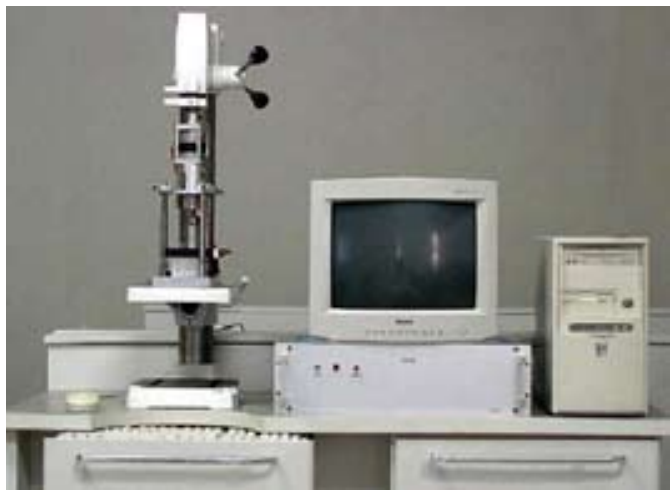

Figura - 2 Cicladora Mecânica Artesanal 


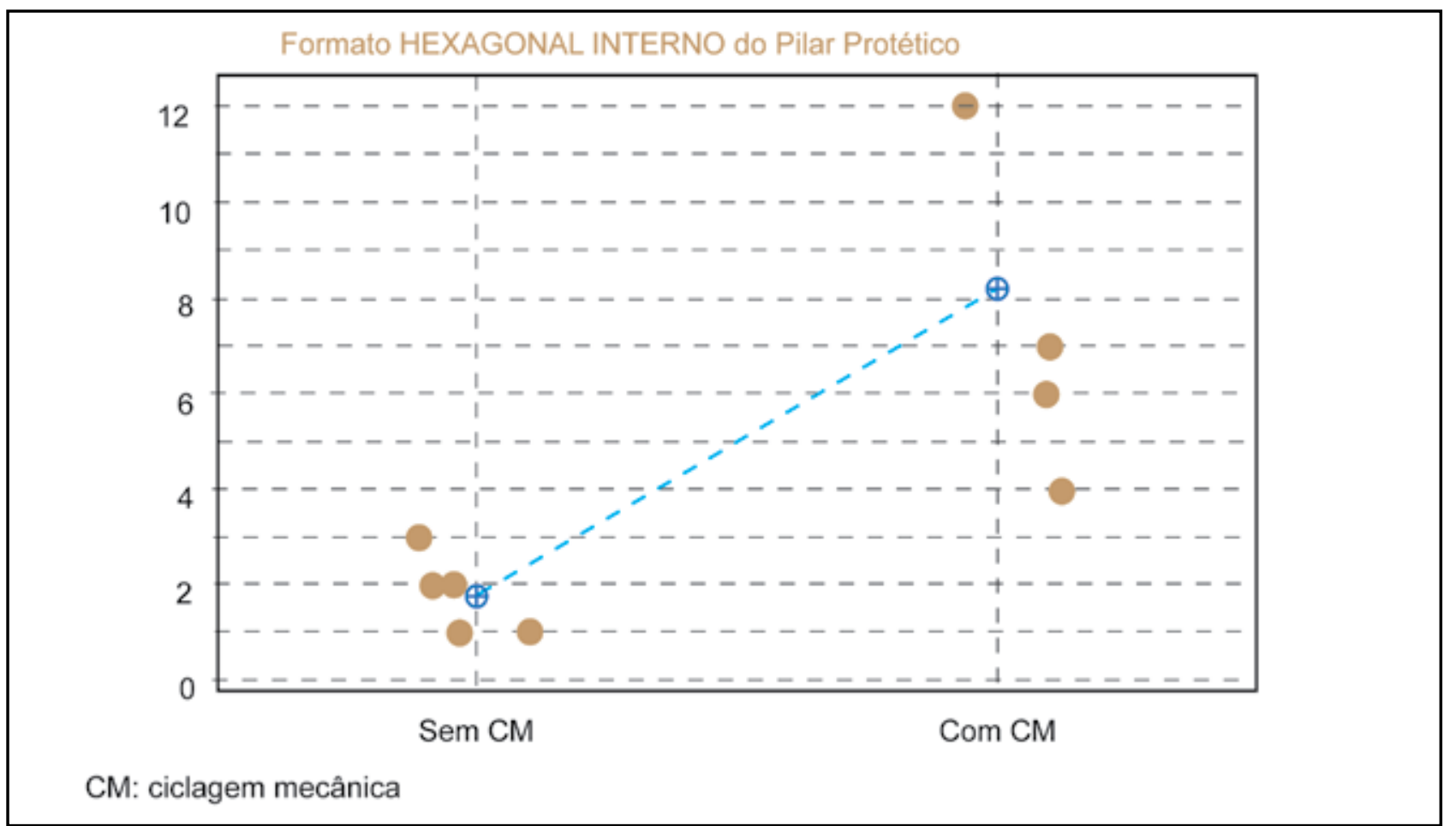

GRÁFICO 1 - Gráfico de pontos ao redor da média, referente aos valores de perda de torque registrados nas amostras com HI, segundo a condição Ciclagem Mecânica.

\section{Dıscussão}

A metodologia empregada neste estudo simulou a condição clínica de estabilidade da união entre componentes protéticos e o implante em função na cavidade bucal a curto prazo, o que equivaleria às primeiras semanas após a instalação da prótese sobre o implante. Este é um fator relevante, visto que a reprodução das condições observadas clinicamente, permite a realização de considerações em virtude da aplicabilidade clínica dos implantes e seus componentes protéticos.

Os resultados obtidos indicam que, embora se tenha utilizado um torque inicial considerado ideal, obedecendo as instruções do fabricante, a perda da força de união entre as estruturas avaliadas foi elevada. Dados oscilaram entre $29 \mathrm{~N}$ e $18 \mathrm{~N}$ de torque aplicado na remoção dos parafusos, tendo sido encontrados os menores valores no grupo que foi submetido à ação de ciclagem mecânica. Esses valores são relativamente baixos em relação ao torque utilizado inicialmente. Esse resultado é sugestivo de uma micromovimentação na interface entre pilar protético e implante, o que pode ser um potencial de falha nas próteses ${ }^{16} \mathrm{e}$ de irritação mecânica dos tecidos moles adjacentes ao implante ${ }^{16,18}$, se considerarmos condições clínicas

O número de ciclos aplicados em cada amostra desse estudo, 50000 ciclos, equivale a um período de tempo estimado, em função clínica, de aproximadamente 20 dias, baseando-se na suposição de que um indivíduo tem 3 episódios de mastigação por dia, cada um com 15 minutos de duração em uma razão de 60 ciclos por minuto $(1 \mathrm{~Hz})^{16}$. Esse indicador é preocupante, já que sugere um desajuste do componente protético nesse estreito período, o que pode ser, a longo prazo, um problema. A possibilidade de que tenha havido deslocamento das estruturas é muito grande. Análise pela MEV da interface implante-pilar em amostras antes e depois da ciclagem mecânica mostrou queda na qualidade da adaptação dos componentes após o ensaio ${ }^{19}$. Ainda, uma avaliação que simulou a perda de $1 / 4$ de volta, por ajustes laboratoriais em parafusos de fixação submetidos posteriormente à ciclagem mecânica, mostrou grande desajuste na interface pilar-implante, que saltou de uma média de $14 \mu \mathrm{m}$ para valores acima de $50 \mu \mathrm{m}$, o que indica que realmente 
há diminuição na vida útil dos pilares parafusados em função ${ }^{20}$.

Os estudos mostram performances variáveis dessa união implante-pilar, mas o ponto mais focado é um aspecto particular do sistema, incluindo a comparação dos métodos de apertamento do parafuso, torque requerido para desadaptação depois de simulada a função, rotação inadequada, entre outros ${ }^{16}$. O critério adotado pela presente pesquisa está dentro das variáveis que são consideradas, a influência da ação mecânica na estabilidade de tais estruturas, sendo, portanto, um fator relevante.

A perda elevada do torque inicial observada no período avaliado nesse estudo pode ser indicativa de considerável desajuste na interface, fator preocupante a longo prazo, contudo um estudo mostra que a perda de ajuste é maior nos períodos iniciais e tende a diminuir com o passar do tempo. De 107 implantes avaliados, 34 apresentaram falhas, 17 ocorreram na primeira semana, 7 em um mês, 5 em seis meses e 5 em um $\mathrm{ano}^{21}$. Ainda, em outra pesquisa, os resultados mostram que há uma condição satisfatória da estabilidade da união pilar-implante em amostras que receberam carga inicial de 30 N.cm durante 2 a 3 anos em função clínica, sem necessidade de reajustes ${ }^{22}$.

$\mathrm{O}$ torque inicial utilizado nessa pesquisa respeita as instruções do fabricante. Um estudo sugere que o uso de uma carga inicial maior que a indicada pode permitir estabilidade superior na união e menor desajuste sem aparente comprometimento do sistema ${ }^{16}$.
Nesta pesquisa, no grupo que recebeu o valor de torque inicial ideal, de 32N.cm, houve alteração de cerca de $40 \%$ na interface das amostras, enquanto que no grupo com valor superior, de $48 \mathrm{~N} . \mathrm{cm}$, a alteração ocorreu em cerca de $32 \%$ das amostras. Contudo, outro estudo ressalta que a carga indicada pelo fabricante é cerca de $75 \%$ do limite de resistência do parafuso de fixação, dessa forma, cargas adicionais podem provocar deformações plásticas e o parafuso pode desajustar, o que pode conduzir a falha potencial do dispositivo ${ }^{19}$. Indica também como alternativa para diminuir os problemas na interface implante-pilar o uso de uma plataforma mais ampla, que promoveria estabilidade superior e diminuiria a carga sobre o parafuso (implantes de $5 \mathrm{~mm}$ suportaram cargas até $79 \%$ maiores que os implantes com $4 \mathrm{~mm})$.

\section{CONCLUSÃO}

O torque de remoção dos parafusos de fixação de pilares protéticos, em qualquer uma das condições avaliadas (com ou sem ação da ciclagem mecânica) é menor que o torque inicial utilizado para sua colocação.

Os pilares protéticos que receberam a da ciclagem mecânica tiveram perda estatisticamente significante em relação ao torque de remoção sem ciclagem.

Tais dados indicam a necessidade de acompanhamento clínico das próteses unitárias sobre implantes e que reajustes periódicos dos pilares protéticos podem ser necessários.

\begin{abstract}
The success in the prosthetic rehabilitation treatment on bone-integrated implants can be compromised by mechanical imperfections on its components, such as misalignment and even though breaking of the prosthetic component when displayed to the chewing function. The present study evaluated, in vitro, the misalignment occurred between implant and abutment using the gauging and comparison of the initial the torque required to remove the retention screws of the abutments in implants with internal hexagon, with regard to the necessary torque removal of these structures before and after the simulation of the chewing function. Tests of cyclic loading had been induced on the prosthetic abutments and the torque removal of the screws was measured. There were significant reduction in the values of torque removal of all the samples, and the loss was still bigger in the samples submitted to the cyclic loading tests. The gotten data allow to assume that there is, always, reduction in the values of torque removal of these components and that the chewing function increases the probability of misalignment in the joint implant-abutment.
\end{abstract}

\title{
UNITERMS
}

Implants; prosthetic component; cyclic loading test; torque removal. 


\section{RefERÊnCIAS}

1. Dias AH et al. A influência da ciclagem mecânica na infra-estrutura cerâmica-revestimento cerâmico. PGR Facul Odontol SJC 2002. 5(1): 64-68.

2. Moraes LMC. Avaliação da adaptação vertical entre intermediários do tipo esteticone e cilindros pré-usinados variando as condições: sobrefundição, torques e parafusos de fixação. Bauru; 2003. [Tese de Doutorado - Faculdade de Odontologia de Bauru - USP].

3. Lima VJM. Estudo comparativo da resistência a flexão e das estruturas metalúrgicas do titânio grau I e IV através de diferentes processos de fundição, utilizados na confecção de próteses sobre implantes ósseointegrados [Tese de Doutorado]. São José dos Campos: Faculdade de Odontologia de São José dos Campos, - UNESP; 2001.

4. Bonachela WC. Avaliação da adaptação da interface intermediários/ cilindro de plásticos fundidos em titânio e $\mathrm{Co}-\mathrm{Cr}$, antes e após a soldagem a laser variando os parafusos de fixação (fenda e hexágono) [Tese de Doutorado]. Bauru: Faculdade de Odontologia de Bauru, USP; 2002.

5. Brosco HB. Precisão da adaptação de intermediários transmucosos unitários em sistemas nacionais de implantes compatíveis com hexágono externo Bauru; 2001. [Tese de Doutorado - Faculdade de Odontologia de Bauru - USP].

6. Binon PP. Implants and components:entering the new millenium. Int J Oral Maxx Impl 2000. 15:76-9.

7. Elias CN, Figueira DC. Torque para soltar parafusos de próteses sobre implante. RBO 2003. 60(5):343-345.

8. Alkan I, Sertgöz, Ekici B. Influence of occlusal forces on stress distribution in preloaded dental implant screws. J Prosthet Dent 2004. 91(4): 320-325.

9. Cibirka RM, Nelson SK, Lang BR, Rueggeberg FA. Examination of the implant-abutment interface after fatigue testing. J Prosthet Dent 2001. 85(3):268-75

10. Pesun IJ, Brosky ME, Korioth TW, Hodges J, Devoe BJ. Operatorinduced compressive axial forces during implant gold screw fastening. J Prosthet Dent 2001. 86(1): 15-19.

11. Jacques LB. Análise do estresse gerado em componentes de prótese fixa implanto-suportada, através do uso de extensômetros. Bauru; 2000. [Tese de Doutorado - Faculdade de Odontologia de Bauru - USP].

12. Kano, SC, Bonfante G, Hussne R, Siqueira AF. Use of base casting alloys for implant framework: marginal accuracy analysis. J Appl Oral Sci 2004; 12(4): 337-343.
13. Dellow AG et al. Interfacial Fit of For Dental Implant Systems. Int $\mathbf{J}$ Prosth 1997, 10(3): 216-221.

14. Itinoche MK, Oyafuso DK, Miyashita E, Araújo MAJ, Bottino MA. Avaliação da influência da ciclagem mecânica na resistência à flexão de cerâmicas. Cienc Odontol Bras 2004; 7(2): 47-54.

15. Itinoche MK. Estudo da influência da ciclagem mecânica na resistência à flexão de cerâmicas. São José dos Campos; 2002. [Tese de Doutorado - Faculdade de Odontologia de São José dos Campos - UNESP].

16. Gratton DG, Aquilino SA, Stantford CM. Micromotion and dynamic fatigue properties of the dental implant-abutment interface. J Prosthet Dent 2001; 85(1): 47-52.

17. Castelnuovo J, et al. Fracture load and mode of failure of ceramic veneers with different preparations. J Prosthet Dent 2000, 83(2): 171179

18. Krejci I, Mueller E, Lutz F. Effects of thermocycling and occlusal force on adhesive compocite crowns. J Dent Rest 1994; 73(6):1228-32.

19. Boggan RS, Strong JT, Misch CE, Bidez MW. Influence of hex geometry and prosthetic table width on static and fatigue strength of dental implants. J Prosthet Dent 1999; 82(4): 436-40.

20. Hoyer AS, et al. Dynamic fatigue properties of the dental implantabutment interface:Joint opening in wide-diamanter versus hex-type implants. J Prosthet Dent 2001; 85(6): 599-607.

21. Jemt T, Laney WR, Harris D, Henry PJ, Krough PH Jr, Polizzi G, et al Osseointegrated implants for single tooth replacement: a 1-year report from a multicenter prospective study. Int J Oral Maxillofac Implants $1991 ; 6: 29-36$.

22. Dixon DL, Breeding LC, Sadler JP, McKay ML. Comparison of screw loosening, rotation, and deflection among three implants designs. J Prosthet Dent 1995. 74(3):270-8.

Recebido em 14/09/07 Aprovado em 26/10/07

Correspondência: Patrícia Maria Rangel

Rua Diamantina, 83

12220-663 - São José dos Campos - SP - Brasil e-mail: patymrangel@hotmail.com 